\title{
Review Effect of Caffeine Overdose
}

\author{
Zaheer Ahmed Chandio ${ }^{1, *}$, Aaisha Sidiqua1', Mazhar Iqbal Khaskheli², Amrat Waghani'1, Wazir Ali Metlo \\ 1Department of Chemistry, Shaheed Benazir Bhutto University, Shaheed Benazirabad, Pakistan. \\ 2M. A. Kazi Institute of Chemistry, University of Sindh, Jamshoro, Pakistan. \\ ${ }^{3}$ Department of Molecular Biology and Genetics, Shaheed Benazir Bhutto University, Shaheed Benazirabad, Pakistan.
}

\section{ABSTRACT}

Background: Caffeine is from methylxanthine class which mainly stimulates the central nervous system. Caffeine is one of the most widely used psychoactive drugs in the world. It is commonly found in beverages, chocolates, cocoa containing products and in medications. Caffeine is recognized as legal and its consumption is unregulated all over the world.

Objectives: The objective of this study is to summarize the impact of caffeine on behavioral and health alterations, in both controlled and overdose conditions. Moreover, the specifics of caffeine withdrawal and a number of guidelines on how to handle reducing or quitting caffeine intake altogether is also highlighted.

Methodology: To compose this review, more than seventy research and review articles were overviewed that were published over a period of the last twenty years, using Google Scholar search engines.

Results: Caffeine regular use causes physical dependence which may become the caffeine withdrawal sign that can consequently harm normal working. The most important function of caffeine is that it can reversibly block the adenosine performance on its receptor and as a result which prevents the beginning of drowsiness encouraged by adenosine. Caffeine also stimulates certain portions of the autonomic nervous system symptom including fatigue, drowsiness, depressed mood, headache, difficulty concentrating, decreased energy, decreased contentedness, decreased alertness, irritability and unclear headed.

Conclusion: It is concluded that utilization of caffeine in a prescribed dose can have a good impact on health and may decrease addictive symptoms. Additionally, reducing caffeine dosage over a six-week period guide to successful, long-term caffeine cessation with very few side effects.

\begin{tabular}{l} 
Keywords \\
$\begin{array}{l}\text { Caffeine, Beverages, Headache, Fatigue, } \\
\text { Insomnia, Anxiety }\end{array}$ \\
\hline Cite this article: Chandio ZA, Sidiqua A, Khaskheli MI, Waghani A, Metlo WA. Review Effect of \\
Caffeine Overdose. RADS J Biol Res Appl Sci. 2020; 11(2):154-158. \\
\hline This is an Open Access article distributed under the terms of the Creative Commons Attribution License \\
(http://creativecommons.org/licenses/by/4.0), which permits unrestricted use, distribution, and reproduction in \\
any medium provided the original work is properly cited.
\end{tabular}

\section{INTRODUCTION}

Caffeine is considered to be the most widely used psychostimulant drugs throughout the world. Chemically, it is an alkaloid belonging to family methylxanthine. Its chemical and systematic name is 1,3,7-trimethylxanthine. In its pure form, caffeine is white glistening needle or powder, intensely bitter and fleecy ${ }^{1}$. Caffeine can be found in various products, but a majority of caffeine is available in caffeinated drinks, tea, coffee and energy drinks. Caffeine

is naturally found in chocolate but it is also added by the manufacturers to some foods and candies. Medications like flu and cold tablets may also contain some amount of caffeine. Its supplements are easily available in markets in the form of powder or tablets. This form contains far more amount of caffeine as compared to coffee and therefore, it becomes difficult for people to measure how much caffeine they have already taken. Caffeine is also naturally present 
in variable amounts in the leaves, beans and fruits of over sixty plants. Caffeine has turned the entire world to it and is on the top of the list of beverages which are sold regularly ${ }^{2}$. Apart from this regular intake of caffeine, it is considered to be dangerous for humans. Taking a cup of coffee daily all through the year i.e., for 365 days and testing your blood on the $366^{\text {th }}$ day will measure $3.03 \%$ of caffeine toxins or poisons that could, kill a person if its percentile reaches above $10 \%{ }^{3,4}$.

Caffeine may have both positive and negative health effects. Literature reveals that caffeine plays a very important role in protecting the liver from cancer when consumed in moderate amount ${ }^{5}$. Another review indicated that taking 2-4 cups of coffee regularly reduces the risk of death from almost all the causes. Recent studies have connected caffeine use with good effects even on the brain $^{6}$. A study published in 2013, suggested that taking 24 cups of coffee every day can enhance long term memory, furthermore, it can also decrease suicide risk in adults who consume a moderate amount of caffeine ${ }^{7,8}$. Caffeine containing beverage consumption has been reported to be associated with reduced bone mass and increased fracture risk in some, but not most, observational studies ${ }^{9}$. A study also claims that regular caffeine intake can protect an individual against type 2 diabetes, cardiovascular disease, stroke and Parkinson's disease ${ }^{10}$. On the other hand, ingesting a high amount of caffeine may lead to more adverse health effects. The results of a study suggested that using $300 \mathrm{mg}$ of caffeine per day during pregnancy can raise the risk of a baby having a low birth weight ${ }^{11}$. An article identified lots of unfavourable effects when consumption of caffeine is over $400 \mathrm{mg}$ per day, including anxiety, sleeping problems, fast heartbeat, restlessness, agitation and tremors ${ }^{12}$. The lethal dose of caffeine for adults has long been estimated to be approximately $10 \mathrm{~g}$. Characteristic symptoms preceding death in confirmed cases of caffeine fatality include abdominal pain, vomiting, markedly increased heart rate, cardiac arrhythmia, agitation, and seizures. Risk of fatal and non-fatal harm due to caffeine poisoning is increased by several characteristics of the drug and the circumstances surrounding its use, including its generally unregulated availability to children and adults alike, and rapid absorption and distribution within the body. Additionally, caffeine effects are dose-dependent when ingested in amounts that exceed typical dietary levels, and its effects may be additive or synergistic when ingested concomitantly with a range of other compounds including pharmaceuticals and recreational drugs ${ }^{13}$. The reports of death caused by caffeine high dose are usually ventricular fibrillation. This situation occurs if lower chambers of heart vibrate rather than contracting frequently. During ventricular fibrillation, the heart stops beating from average, which results in cardiac arrest ${ }^{14}$.

In another review, a researcher reported 92 deaths from caffeine overdose ${ }^{15}$. It was believed that one-third of these deaths were probably suicidal. Usually, caffeine affects the body when its concentration is more than $15 \mathrm{mg} / \mathrm{L}$ in the blood. However, if the amount exceeds 80 to $100 \mathrm{mg} / \mathrm{L}$ it could be deadly ${ }^{16}$. Overdose of caffeine usually occurs by taking caffeine tablets or dietary supplement rather than taking coffee; mainly people combine these products with coffee, energy drinks or sodas ${ }^{17}$. A supplement raises the risk because caffeine levels become higher than foods and drinks. Consumption of purified caffeine is highly dangerous and is likely to cause the increase in blood caffeine level. The US FDA warns that one teaspoon of powdered caffeine is almost equivalent to twenty-eight cups of coffee. The FDA stresses that highly concentrated and pure caffeine products can cause serious health hazards.

A question arises why do people usually get addicted to caffeine? Caffeine belongs to methylxanthines which is structurally similar to tryptophan (Try). Tryptophan is considered to be a neurotransmitter secreted in cases such as orgasm, when a person is in love, hallucinated to be happy or in coitus ${ }^{18,19}$. Therefore, when a person generally takes chocolate or caffeine, they also usually contain some amount of tryptophan and which acts as a precursor to serotonin. Serotonin is a known neurotransmitter which has the mood-enhancing property whilst high serotonin levels cause the elation effects. Phenylethylamine is a derivative of amphetamine, which is identified for love and attraction, therefore, these are also released when chocolates and caffeine are ingested ${ }^{20}$. 


\section{SYMPTOMS OF CAFFEINE OVERDOSE}

Headache

People have been using caffeine for the treatment of migraine and common pain from a long time because of its unique ability to decrease blood flow, particularly in the brain. Though, consuming caffeine frequently may also cause a headache. Quitting or reducing caffeine after long term use can cause severe migraine-like headache in some persons. Because it is both soluble in water and fat caffeine quickly crosses the blood-brain obstruction where it contracts the blood vessels. Narrowing the blood vessels may cause a reduction in blood flow, which is very helpful to overcome migraine pain. Quitting or reducing caffeine dose rapidly will let the blood vessels to grow unexpectedly, which an increase in the blood flow. This sudden striking increase in blood flow may cause severe pain, throbbing headaches just like in the case of migraine. Such headaches are caused because of the withdrawal of caffeine and can vary in time length and severity. Usually, people use caffeine for the treatment of such headaches; however, it should be kept in mind that they do not use more caffeine than previously consumed. Headaches will be subsided gradually as the brain gets used to the change in the blood flow.

\section{Fatigue}

Mostly in the morning, people use caffeine to improve their alertness levels. By blocking adenosine receptors in the brain, caffeine prevents fatigue and enhances alertness. Adenosine is a neurotransmitter which slows down the central nervous system when the body gets ready to sleep. Nevertheless, when someone rapidly stops or lowers the caffeine dosage, they can have temporarily reverse effect and which feels more tiredness throughout the day. A person can overcome this feeling of tiredness during the day by enhancing his/her sleep throughout the night.

\section{Changes in Mood}

Regularly, low consumption of caffeine may also cause alertness in mood and also diminishes feelings like anxiety. However, using reasonable to high level dosage of caffeine may cause feelings like jitteriness, nervousness and anxiety ${ }^{21}$. Such mood changes are caused because of the effect which caffeine has on a variety of neurotransmitters. These include norepinephrine, glutamate and dopamine. Dopamine has the characteristics of activating pleasure centers which are present in the brain and responsible for controlling behaviours and emotions. A study showed that caffeine use is not only stimulating dopamine production, but it can increase the available number of dopamine receptors present in the brain, which overall enhance dopamine's effect on the brain ${ }^{22}$. Glutamate supports communication between nerve cells and plays a very important role during learning and memory. The norepinephrine is produced in the brain when a person feels stress or danger in a process known as "fight-orflight" reaction. Norepinephrine boosts breathing rate, heart rate and blood glucose levels. Quickly leaving caffeine may cause drastic effects on the chemical composition in the brain, results in the feelings like depression, anxiety or irritability.

\section{Difficulty in Concentrating}

Caffeine can interact with certain chemicals present in the brain therefore, it can affect concentration level and memory. A study reveals that taking just $80 \mathrm{mg}$ of caffeine dose results in the up-gradation in working memory and provides quick response time in humans ${ }^{23}$. Findings recommend that regular use of caffeine can lower the risk level of dementia or cognitive impairment in old aged women ${ }^{24}$. People usually experience obscurity in concentration when suddenly quitting caffeine intake. In unavailability of caffeine, molecules of adenosine can encourage feelings like fatigue which may affect the capacity to concentrate.

\section{Constipation}

Caffeine stimulates contractions in the intestines and colon. These contractions assist the food to move fast and waste substances through the gastrointestinal tract. The regular consumption of caffeine may face mild constipation when they reduce the caffeine dose. People can prevent this problem by regularly taking fiber rich foods and staying hydrated maximum level ${ }^{25}$. 


\section{CONCLUSION}

People can be able to prevent caffeine withdrawal symptoms by slowly reducing their caffeine dose over time. Progressively reducing caffeine dosage over a six-weeks period guides to successful, long-term caffeine cessation with very few side effects. Caffeine quitting may cause dramatic changes to brain chemistry, which may affect a cognitive ability and a person's mood. People who regularly drink coffee can slowly decrease their caffeine dosage by mixing some amount of decaf into their coffee. Those people who take multiple cups can aim to replace one or more with decaf. Maximum sleep also supports to fight against fatigue and feeling well-rested will also support to minimize the body's reliance on caffeine. The remaining hydrated entire day is necessary since headaches and fatigue are also experienced due to dehydration.

\section{CONFLICTS OF INTEREST}

None.

\section{FUNDING SOURCE}

None.

\section{ACKNOWLEDGEMENTS}

This review was supported by the Department of Chemistry, Shaheed Benazir Bhutto University, Shaheed Benazirabad, Pakistan. We are thankful to our colleagues who provided expertise that greatly assisted the review.

\section{LIST OF ABBREVIATIONS}

FDA Food and Drug Administration

Try Tryptophan

\section{REFERENCES}

1. Caballero B, Finglas $P$, Toldra F. Encyclopedia of food and health. Elsevier Sci. 2015; 561-9.

2. Celine MR, Marilyn CC. Caffeine in the diet: Countrylevel consumption and guidelines. Nut. 2018; 10(11):1772-85.

3. Barasch A, Gordon SC. Effects of caffeine on salivation. Oral Health Case Rep. 2016; 1(107):7-10.

4. Morphett L. A case study investigating a behavioral intervention to reduce caffeine consumption. J Women's Health Care. 2014; 3:186-93.
5. Inoue M, Tsugane S. Coffee drinking and reduced risk of liver cancer: Update on epidemiological findings and potential mechanisms. Curr Nutr Rep. 2019; 8(3):1826.

6. Bolton S, Null G. Caffeine: Psychological effects. Use and abuse. Orthomol Psych. 1981; 10(3):202-11.

7. Michael B, Andrea P, Emanuel S, Peter CW, Jules $A$, Hans-Jürgen M. World Federation of Societies of Biological Psychiatry (WFSBP) guidelines for biological treatment of unipolar depressive disorders, Part 1: Update 2013 on the acute and continuation treatment of unipolar depressive disorders. World $\mathrm{J}$ Biol Psych. 2013; 14-25.

8. Nehlig A. Is caffeine a cognitive enhancer? J Alzheimer's Dis. 2010; 20(1):85-94.

9. Heaney RP, Effects of caffeine on bone and the calcium economy. Food Chem Toxicol. 2002; 40:126370.

10. Qi H, Li S. Dose-response meta-analysis on coffee, tea and caffeine consumption with risk of Parkinson's disease. Geriat Gerontol Int. 2014; 14(2):430-9.

11. Jahanfar SJ, Sharifah H. Cochrane Pregnancy and Childbirth Group (ed.). Effects of restricted caffeine intake by mother on fetal, neonatal and pregnancy outcomes. Cochrane Database Sys Rev. 2015; (6):5372.

12. Simone C, Piacentino D, Gabriele S, Mariarosaria A. Caffeine: Cognitive and physical performance enhancer or psychoactive drug? Curr Neuropharmacol. 2015; 13(1):71-88.

13. James EJ. Death by caffeine: How many caffeine related fatalities and near misses must there be before we regulate? J Caff Res. 2012; 2:149-52.

14. Simone $C$, Daria $P$, Vittorio $F$, Paola F, Luigi $C$, Mariarosaria A. Caffeine-Related Deaths: Manner of Deaths and Categories at Risk. Nutrients. 2018; 10(5):611-8.

15. Willson $\mathrm{C}$. The clinical toxicology of caffeine: A review and case study. Toxicol Rep. 2018; 5:1140-52.

16. Higdon JV, Frei B. Coffee and health: A review of recent human research. Crit Rev Food Sci Nutr. 2006; 46:101-23.

17. Nawrot $P$, Jordan $S$, Eastwood J, Rotstein J, Hugenholtz A, Feeley M. Effects of caffeine on human health. Food Addit Contam. 2003; 20:1-30. 
18. Ruenis AP. Effects of caffeine and theophylline on the development of dental caries in rats. Biol Pharm Bull. 2000; 23:339-43.

19. Lagerlof F. Intra and inter-individual differences in salivary sucrose clearance over time. Caries Res. 2004; 28:348-52.

20. Lane SD. Comparison of caffeine and d-amphetamine in cocaine-dependent subjects: Differential outcomes on subjective and cardiovascular effects, reward learning, and salivary paraxanthine. J Addict Res Ther. 2014; 5:176-83.

21. Nehlig A, Daval JL, Debry G. Caffeine and the central nervous system: Mechanisms of action, biochemical, metabolic and psychostimulant effects". Brain Res Rev. 1992; 17(2):139-70.

22. Volkow ND, Wang GJ, Logan J, Alexoff D, Fowler JS, Thanos PK, et al. Caffeine increases striatal dopamine D2/D3 receptor availability in the human brain. Transl Psychiatry. 2015; 5:1-15.
23. Sarah B, Brian T, Andrew S. Attentional and working memory performance following alcohol and energy drink: A randomised, double-blind, placebo-controlled, factorial design laboratory study. PLoS One. 2019; 14(1):1-13.

24. Ira D, Sally AS, Beverly MS, Karen LM, JoAnn EM, Mara ZV, et al. Relationships between caffeine intake and risk for probable dementia or global cognitive impairment: The women's health initiative memory study. J Gerontol Biol Sci Med Sci. 2016; 71(12):1596-602.

25. Sloots CEJ, Felt-Bersma RJF, West RL, Kuipers EJ. Stimulation of defecation: Effects of coffee use and nicotine on rectal tone and visceral sensitivity. Scan J Gastroenterol. 2005; 40: 808-13. 